\title{
Entanglement Identification of Arbitrary Two-Qubit Quantum Channels and the Capabilities to Realise Quantum Teleportation
}

\author{
Lila Yuwana, Agus Purwanto, Endarko and Dwi Januriyanto \\ Department of Physics, Institut Teknologi Sepuluh Nopember, Surabaya, Indonesia \\ lila@physics.its.ac.id, purwanto@physics.its.ac.id, endarko@gmail.com,dwijanuriyanto91@gmail.com
}

\begin{abstract}
Objectives: To identify the entanglements of arbitrary two-qubit quantum channels and the capabilities to realise quantum teleportation. Methods: The implementation of Schmidt de composition and measurements of quantum system state are equiped in this research. Findings: By constructing the general form of an arbitrary two qubit and measurement state, we able to determine an entanglement of an arbitrary two qubit by utilising Schmidt decomposition. Next, a capability of an arbitrary two qubit entangled state to realise teleportation can be identified by formulating measurement state and unitary operator. Improvement: The methods used in this paper have relatively simple calculations and very beneficial to be implement before developing quantum teleportation system experimentally.
\end{abstract}

Keywords: Entangled States, Quantum Channels, Qubit (Quantum Bit), Quantum Teleportation

\section{Introduction}

The emergence of quantum teleportation in 1993 proposed leads to perseverance to perform research on this topic theoretically and experimentally. ${ }^{1}$ Introduced quantum teleportation of an arbitrary a qubit using an entangled state of Einstein-Podolsky-Rosen (EPR) pair. In experimental aspect, several exceptional experimental implementations of quantum teleportation have been exhibited both in laboratory and in free space. $\frac{2-6}{6}$ On the other side, there is a continuation of research theoretic call yon quantum teleportation. One of interesting topics on quantum teleportation is entangled state. Have successfully formulated two-qubit quantum teleportation system by delivering the requirements of a realisation of teleportation. Moreover, identify a quantum channel whether is able to realise teleportation or not by calculating its determinant of transformation operator. Next, if the determinant of operator is zero, then quantum teleportation cannot be realised. ${ }^{I}$ A year later, showed the relation between the determinant of transformation operator and SLOCC (stochastic local operation and classical communication) invariant also can be used to determine the capability of quantum channels to realise quantum teleportation..$^{-}$Recently, investigated quantum teleportation single qubit with Werner-like state as a resource in 2014 to continue their previous results on quantum teleportation of single-qubit pure state using non-maximally entangled pure state. ${ }^{9}$

However, in the case of teleportation of single-qubit pure state still remains an area that could be explored. This is because the realisation of quantum teleportation also depends on entangled state which is used as quantum channel. In this paper, we report entangled states that are able and not able to realise quantum teleportation. We deployed Schmidt Decomposition to define the entanglements of quantum channels. Furthermore, we determine the capability of quantum channels to realise quantum teleportation by using particular measurements which we have prepared.

*Author for correspondence 


\section{Single-Qubit Teleportation through EPR States}

Suppose Alice delivers information Bob in the form of unknown single-quit state (particle 1$)^{1}$

$|\chi\rangle_{1}=a|0\rangle_{1}+b|1\rangle_{1}$

where

$|a|^{2}+|b|^{2}=1$.

Generally, the information $|\chi\rangle$ is sent to Bob by sharing one of entangled state EPR pairs/Bell states (particle 2 and 3)

$\varphi_{23}^{1}=\frac{1}{\sqrt{2}}(|00\rangle+|11\rangle)_{23} ; \varphi_{23}^{2}=\frac{1}{\sqrt{2}}(|00\rangle-|11\rangle)_{23}$

$\varphi_{23}^{3}=\frac{1}{\sqrt{2}}(|01\rangle+|10\rangle)_{23} ; \varphi_{23}^{4}=\frac{1}{\sqrt{2}}(|01\rangle-|10\rangle)_{23}$,

where subscript 1, 2, and 3 in Equation (1) and (3) denote the possessor of particle. It means particle 1 in $|\chi\rangle$ belongs to Alice and particle 2 and 3 in $\left|\varphi^{n}\right\rangle$ are possessed by Alice and Bob. In order to show a process of quantum teleportation, it is beneficial to put one of EPR states to be a quantum channel, for example, we have selected $\left|\varphi_{23}^{3}\right\rangle$ in Equation (3).

Theoretically, the system state of three particles is $|\Psi\rangle_{123}=|\chi\rangle_{1} \otimes|\varphi\rangle_{23}$

$=\frac{1}{\sqrt{2}}(a|001\rangle+b|101\rangle+a|010\rangle+b|110\rangle)$

By combining Alice's state and the quantum channel yields annihilation of initial state of Alice which means this teleportation system is in accordance with the minimum requirement of quantum teleportation system, i.e. non-cloning theorem..$^{10}$

Next, Alice employs a measurement on the system state by interacting with another quantum state, for example one of EPR state listed in Equation (3).

To show a calculation of measurement on the system state (3), we selected $\left|\varphi_{23}^{1}\right\rangle$ to measure the system and to be symbolised as

$|\pi\rangle=\frac{1}{\sqrt{2}}(|00\rangle+|11\rangle)$.

The mechanism to teleport the information to Bob is

$\left.\left.\left.(\langle\pi| \otimes I)\right|^{\Psi}\right\rangle_{123}=\left.\langle\pi \mid \Psi\rangle_{12} \otimes I\right|^{\top}\right\rangle_{3}$.
Obviously, quantum state that received by Bob is

$|\Psi\rangle_{3}=\frac{1}{2}(a|1\rangle+b|0\rangle)$

That is not completely the same as initial state in Equation (1). The consequence of this is we have to implement a suitable unitary operator to reconstruct quantum state in Equation (7) to be exactly the same as Equation (1).The appropriate unitary operator is $2 \sigma_{x}$, where $\sigma_{x}$ is selected Pauli matrix. By applying $2 \sigma_{x}$ to Equation (7), we have

$|\Psi\rangle_{3}=2 \sigma_{x} \frac{1}{2}(a|1\rangle+b|0\rangle)=(a|0\rangle+b|1\rangle)_{3}$.

Eventually, Bob receives the quantum state that is the same as initial state sent by Alice.

For other EPR pairs in Equation (3) aplied as a measurement, the related unitary operators implemented to Bob's quantum states are

- For measurement $|\pi\rangle=\varphi_{23}^{2}$,

$|\Psi\rangle_{3}=2 \sigma_{x} \sigma_{z} \frac{1}{2}(a|1\rangle-b|0\rangle)_{3}=(a|0\rangle+b|1\rangle)_{3}$

- For measurement $|\pi\rangle=\varphi_{23}^{3}$,

$|\Psi\rangle_{3}=2 I \frac{1}{2}(a|0\rangle+b|1\rangle)=(a|0\rangle+b|1\rangle)_{3}$

- For measurement $|\pi\rangle=\varphi_{23}^{2}$,

$|\Psi\rangle_{3}=2 \sigma_{z} \frac{1}{2}(a|0\rangle-b|1\rangle)=(a|0\rangle+b|1\rangle)_{3}$

where

$I=\left(\begin{array}{ll}1 & 0 \\ 0 & 1\end{array}\right), \sigma_{x}=\left(\begin{array}{ll}0 & 1 \\ 1 & 0\end{array}\right), \sigma_{y}=\left(\begin{array}{cc}0 & -i \\ i & 0\end{array}\right), \sigma_{z}=\left(\begin{array}{cc}1 & 0 \\ 0 & -1\end{array}\right)$

By implementing one of the unitary operators in Equation (9) for selected measurement, Bob obtain particle 3 which is really the same as initial state delivered by Alice.

\section{Quantum Channel and Measurement using Two-Qubit in General Form}

A realisation of quantum teleportation depends on delivered quantum state, quantum channel, and measurement performed by sender (Alice). If Alice send single-qubit in Equation (1) and we arrange general form of two-qubit quantum channel

$|\varphi\rangle_{A B}=c_{0}|00\rangle+c_{1}|01\rangle+c_{2}|10\rangle+c_{3}|11\rangle$

and general formulation of measurement

$|\pi\rangle=m_{0}|00\rangle+m_{1}|01\rangle+m_{2}|10\rangle+m_{3}|11\rangle$

We have the general form of system state is 


$$
\begin{aligned}
|\Psi\rangle= & |\chi\rangle_{A} \otimes|\varphi\rangle_{A B} \\
= & x_{0} c_{0}|000\rangle+x_{0} c_{1}|001\rangle+x_{0} c_{2}|010\rangle+x_{0} c_{3}|011\rangle \\
& +x_{1} c_{0}|100\rangle+x_{1} c_{1}|101\rangle+x_{1} c_{2}|110\rangle+x_{1} c_{3}|111\rangle
\end{aligned}
$$

Next, we perform Alice's measurement by interacting Equation (13) with $(\langle\pi| \otimes I)$ of Equation (12) to eliminate particle 1 and 2 . As a result, Bob receives the information from particle 3

$$
\begin{aligned}
& \left|\chi^{\prime}\right\rangle_{B}=\left(m_{0} x_{0}+m_{2} x_{1}\right)\left(c_{0}|0\rangle_{3}+c_{1}|1\rangle_{3}\right)+\left(m_{1} x_{0}+m_{3} x_{1}\right) \\
& \left(c_{2}|0\rangle_{3}+c_{3}|1\rangle_{3}\right) .
\end{aligned}
$$

Finally, we applies a unitary operator according to Alice's measurement to rebuilt Equation (14) to be the same as Alice's state that have delivered.

\section{Arbritary Two-Qubit Quantum Channel and Measurement}

In the previous example, we used EPR pair to represent quantum channel and measurement. It does not require work to prove the entanglement of quantum channel and measurement because EPR pairs are proven to be entangled state and able to realise quantum teleportation.

On the other hand, the using of an arbitrary two-qubit demands methods to verify that an arbitrary two-qubit is entangled state or not. Moreover, although an arbitrary two qubit is entangled state, it requires calculation to verify its capability to realise quantum teleportation. We employ Schmidt decomposition to identify an entanglement of an arbitrary two-qubit quantum channel. .0 .11 Consecutively, for an entangled two-qubit need to be authenticate its capability to realise quantum teleportation by formulating two-qubit measurement. If there is no suitable measurement, then the entangled two-qubit is not able to realise quantum teleportation.

Next, we provide the calculations to show the process of two-qubit entaglement validations and to determine appropriate measurements for verifiable entangled states. For first example, suppose we have quantum channel

$|\varphi\rangle=\frac{1}{\sqrt{2}}(|00\rangle+|11\rangle)$

that will be verified whether it is entangled state or not. We use Schmidt decomposition to examine the entanglement. According to Schmidt decomposition, coefficients of Equation (15) form a matrix $\underline{10,11}$ $c=\left(\begin{array}{cc}\frac{1}{\sqrt{2}} & 0 \\ 0 & 0\end{array}\right)$,

where

$c^{\dagger} c=\left(\begin{array}{ll}1 & 0 \\ 0 & 0\end{array}\right)$.

Now, the eigen value of Equation (17) is equal to zero

$\left|c^{\dagger} c-I \lambda\right|=0$

$\left|\begin{array}{cc}1-\lambda & 0 \\ 0 & -\lambda\end{array}\right|=0$

$-\lambda(1-\lambda)=0$

Now, we have two result of $\lambda$, i.e. $\lambda_{1}=1$ and $\lambda_{2}=0$. As a result, the matrix of the eigen values of $c^{\dagger} c$ is

$\Lambda=\left(\begin{array}{cc}\sqrt{1} & 0 \\ 0 & 0\end{array}\right)$

Based on Schmidt decomposition, if the number of nonzero diagonal components is greater than one, then the quantum channel is entangled. On the other hand, if the number of non-zero diagonal components is only one, then the quantum channel is separable. As a consequence, we terminate the calculation.

Next example, we employ quantum channel as follows

$|\varphi\rangle=\frac{1}{2}(|00\rangle+|01\rangle+|10\rangle-|11\rangle)$

Similar as the previous example,

$c=\left(\begin{array}{cc}1 & 1 \\ 1 & -1\end{array}\right), c^{\dagger} c=\frac{1}{2}\left(\begin{array}{ll}1 & 0 \\ 0 & 1\end{array}\right)$,

$\left|c^{\dagger} c-I \lambda\right|=0$

$\left|\begin{array}{cc}0.5-\lambda & 0 \\ 0 & 0.5-\lambda\end{array}\right|=0$

$(0.5-\lambda)^{2}=0$.

Then we have $\lambda_{1}=\lambda_{2}=\frac{1}{2}$ which form a matrix

$\Lambda=\left(\begin{array}{cc}\frac{1}{\sqrt{2}} & 0 \\ 0 & \frac{1}{\sqrt{2}}\end{array}\right)$.

Equation (21) reveals that the quantum channel is entangled, because the number of non-zero diagonal coefficients is greater than one. It means the next process, which is the verification to determine wheter the quantum channel can realise teleportation or not, need to be executed. From Eq. (19), we have $c_{0}=c_{1}=c_{2}=-c_{3}=\frac{1}{2}$. By utilising Equation (11), (12), (13) and (14) for $m_{0}=m_{1}=m_{2}=-m_{3}=\frac{1}{2}$, we obtain the quantum state possesed by Bob as follows

$\left|\chi^{\prime}\right\rangle_{B}=\frac{1}{2}\left(x_{0}|0\rangle+x_{1}|1\rangle\right)=\sigma_{B}\left(x_{0}|0\rangle+x_{1}|1\rangle\right)$. 
Eventually, we prove that the quantum channel in Equation (19) is able to realise quantum teleportation because there is a appropriate measurement to recontruct Equation (22) to be the same as Equation (1) by operating a unitary operator $\sigma_{B}$. Obviously, from Equation (22) we can determine the associate unitary operator in the form of

$\sigma_{B}=2 I$

Next, let a quantum channel

$|\varphi\rangle=0.5|00\rangle+0.5|01\rangle+0.7|10\rangle+0.1|11\rangle$.

Similar as the previous example,

$c=\left(\begin{array}{ll}0.5 & 0.5 \\ 0.7 & 0.1\end{array}\right), c^{\dagger} c=\frac{1}{2}\left(\begin{array}{cc}0.74 & 0.32 \\ 0.32 & 0.26\end{array}\right)$,

$\left|c^{\dagger} c-I \lambda\right|=0$

$\left|\begin{array}{cc}0.74-\lambda & 0.32 \\ 0.32 & 0.26-\lambda\end{array}\right|=0$

$\lambda^{2}-\lambda+0.09=0$.

Then we have $\lambda_{1}=0.9$ and $\lambda=0.1$ which form a matrix

$\Lambda=\left(\begin{array}{cc}\sqrt{0.9} & 0 \\ 0 & \sqrt{0.1}\end{array}\right)$.

Equation (26) indicates that the quantum channel is entangled, because the number of non-zero diagonal coefficients is greater than one. Now, we determine wheter the quantum channel can realise teleportation or not. From Equation(19), wehave $c_{0}=0.5, c_{1}=0.5, c_{2}=0.7, c_{3}=0.1$ . By utilising Equation (11), (12), (13) and (14) for $m_{0}=m_{1}=m_{2}=-m_{3}=\frac{1}{2}$, we obtain the quantum state possesed by Bob as follows

$\left|\chi^{\prime}\right\rangle_{B}=0.6 x_{0}|0\rangle+0.3 x_{0}|1\rangle-0.2 x_{1}|0\rangle-0.1 x_{1}|1\rangle$

$=\left(0.6 x_{0}-0.2 x_{1}\right)|0\rangle+\left(0.3 x_{0}-0.1 x_{1} \mid 1\right)$.

Unfortunately, Equation (22) state that quantum channel in this example is not able to realise teleportation because there is no unitary operator can transform this equation to be the same as Equation (1). The reason of this is that no measurement state is able to form $\left|\chi^{\prime}\right\rangle_{B}$ to the form of Equation (1).

\section{Conclusion}

The methods of identifying an entanglement of an arbitrary two qubit and its capability to realise teleportation is applied. By examining from the general form of an arbitrary two qubit and measurement state, we have successfully to determine an entanglement of an arbitrary two qubit by utilising Schmidt decomposition. Next, a capability of an arbitrary two qubit entangled state to realise teleportation can be identified by formulating measurement state and unitary operator. If there is no measurement and unitary operator is able to transform measured quantum state, then the entangled state that is used as quantum channel is not able to realise quantum teleportation, and vice versa. Finally, we provide obvious examples to show the calculations.

\section{References}

1. Bennet $\mathrm{CH}$, Brassard G, Crepeau C, Jozza R, Peres A, Wootters WK. Teleporting an unknown quantum state via dual classical and Einstein-Podolsky-Rosen Channels. Physical review letters. 1993; 70(13). Crossref.

2. Boschi D, Branca S, Martini FD, Hardy L, Propescu S. Experimental realization of teleporting an unknown pure quantum state via dual classical and einstein-podolskyrosen channels. Physical review letters. 1998; 80 (6):1121-5. Crossref.

3. Bouwmeester D, Pan JW, Mattle K, Eibi M, Weinfurter H, Zeilinger A. Experimental quantum teleportation. Nature international weekly journal of science. 1997; 390:575-9.

4. Ursin R, Jennewein T, Aspelmeyer M, Kaltenbaek R, Lindenthal M. Quantum teleportation across the Danube. Nature international weekly journal of science. 2004; 430:1-849.

5. Jin XM, Ren JG, Yang B, Yi ZH, Zhou F. Experimental free-space quantum teleportation. Nature Photonics. 2010; 4:376-81. Crossref.

6. Ma XS, Herbst T, Scheidl T, Wang D, Kropatschek S. Quantum teleportation over 143kilometres using active feed-forward. Nature international weekly journal of science. 2012; 489:269-73.

7. Zha XW, Huang CB. The expansion of orthogonal complete set and teleportation of an arbitary two-qubit state. Acta physica sinaca. 2007; 56(4):1875-80.

8. Zha XW, Ren KF. General relation between the transformation operator and an invariant under stochastic local operations and classical communication in quantum teleportation. Physical review A. 2008; 77(1):1-4. Crossref.

9. Verma V, Prakash H. Quantum teleportation of single qubit mixed information state with Werner-like state as resource. In 12th International Conference on Fiber Optics and Photonics; 2014. p. 1-12. Crossref.

10. Nakahara M, Ohmi T. Quantum computing, from linear algebra to physical realization. Boca raton: taylor \& francis Group; 2008. p. 1-440.

11. Nielsen MA, Chuang IL. Quantum Computation and Quantum Information. New York: Cambridge university press; 2010. p. 1-698. Crossref. Crossref. 